\title{
Safety and Putative Benefits of Tracheostomy Tube Placement in Patients on Extracorporeal Membrane Oxygenation: A Single-Center Experience
}

Journal of Intensive Care Medicine I-9

(C) The Author(s) 2019

Article reuse guidelines:

sagepub.com/journals-permissions DOI: I0.II77/08850666/9837939 journals.sagepub.com/home/jic @SAGE

\author{
Jatinder Grewal, MBBS ${ }^{1,2}$, Anna-Liisa Sutt, CPSP, PhD $^{2,3}$, \\ George Cornmell, BSc ${ }^{4}$, Kiran Shekar, MBBS, MD, PhD, FCICM ${ }^{2,3,4,5}$ \\ and John Fraser, MBBS, PhD, FRCP (Glas), FRCA, FFARCSI, FCICM ${ }^{2,3,4}$
}

\begin{abstract}
Purpose: Patients supported with extracorporeal membrane oxygenation (ECMO) have been reported to have increased sedation requirements. Tracheostomies are performed in intensive care to facilitate longer term mechanical ventilation, reduce sedation, improve patient comfort, secretion clearance, and ability to speak and swallow. We aimed to investigate the safety of tracheostomy (TT) placement on ECMO, its impact on fluid intake, and the use of sedative, analgesic, and vasoactive drugs. Methods: Prospective data were collated for all ECMO patients over a 5.5-year period. Data included the cumulative dose of sedatives and analgesics, fluid balance, inotrope and vasopressor requirements, and number of packed red cell (PRC) units transfused. Data were analyzed to determine the differences in the aforementioned between 5 days pre-TT and post-TT insertion. Results: Thirty-one (22.1\%) of 140 patients underwent TT while on ECMO in the study period. Inotrope and vasopressor use was significantly less in the post-TT period compared to pre-TT dose $(P$ value $=.0 \mathrm{I})$. This was in the setting of Sequential Organ Failure Assessment scores the day before TT placement being significantly greater than those on days 2,3 , and 4 . There was a trend toward reduction in analgesic usage in the post-TT period. No major complications of TT were reported. There was no significant difference $(P$ value $=.46)$ in the amount of PRC used post-TT. Conclusions: These data indicate that TT may result in a reduction in vasopressor and inotropic requirement. Data do not suggest increased major bleeding with placement of TT in patients on ECMO. The potential risk and benefits of inserting a TT in ECMO patients need further validation in prospective clinical studies.
\end{abstract}

\section{Keywords}

ECMO, sedation, vasopressor, inotrope, tracheostomy

\section{Introduction}

Extracorporeal membrane oxygenation (ECMO) is an increasingly used modality in intensive care unit (ICU) for patients who have failed traditional respiratory or cardiac supportive therapy. ${ }^{1}$ Use of ECMO has been increasing ${ }^{2}$ despite the lack of robust evidence to support its use. ${ }^{3}$ Some supportive evidence from CESAR trial and encouraging results from use of ECMO during the influenza epidemic of 2009 in Australia ${ }^{2,4-6}$ have certainly played a role in increased uptake of ECMO. Recently published EOLIA trial ${ }^{7}$ has added further support to the use of venovenous (VV) ECMO in respiratory failurewhich seems greater still when assessed in a Bayesian manner. ${ }^{8}$ The optimal mechanical ventilation strategy on ECMO remains debatable, and experienced centers are extubating their patients early and rehabilitating them where feasible. ${ }^{9,10}$ In many others a prolonged period of mechanical ventilation is inevitable, and

\footnotetext{
' Royal Brisbane and Womens Hospital, Brisbane, Queensland, Australia ${ }^{2}$ Critical Care Research Group, Adult Intensive Care Unit, The Prince Charles Hospital and University of Queensland, Brisbane, Queensland, Australia

${ }^{3}$ University of Queensland, Brisbane, Queensland, Australia

${ }^{4}$ Adult Intensive Care Services, The Prince Charles Hospital, Brisbane, Queensland, Australia

${ }^{5}$ Bond University, Gold Coast, Queensland, Australia
}

Received January 13, 2019. Received revised February 14, 2019. Accepted February 25, 2019.

Corresponding Author:

Jatinder Grewal, Royal Brisbane and Womens Hospital, Brisbane, Queensland 4029, Australia.

Email: jpsgdoc@gmail.com 
Journal of Intensive Care Medicine $X X(X)$

these patients may often have increased sedation requirements. ${ }^{11}$ Consequently, this may result in increased fluid and vasoactive drug use, immobilization and neuromuscular weakness, and other adverse consequences of sedation in the ICU. To overcome this, some centers perform a tracheostomy (TT) once patients are stabilized on ECMO, although the risks and benefits of such an approach remain unclear.

While there is no strong evidence for performing an early TT (variably defined in literature between $<4$ and $<10$ days postintubation) $)^{12,13}$ in general critically ill patients, it may still have a role in special patient populations. Tracheostomy is performed within the ICU to facilitate weaning from mechanical ventilation, restore function of the upper airway, continue tracheobronchial toileting, and minimize injury to vocal cords,${ }^{14,15}$ with percutaneous dilatational tracheostomy (PDT) being more common than surgical TT in ICU. ${ }^{16,17}$ Patients with prolonged ventilator requirements (more than 10 days) ${ }^{12}$ often receive a TT. ${ }^{17}$ Reported benefits include decreased sedative drug usage, ${ }^{18}$ shorter ventilation duration,,${ }^{19}$ decreased mortality, ${ }^{12}$ improved patient comfort, improved clearance of secretions, ability to recommence oral intake, and facilitation of communication through the use of a speaking valve. ${ }^{20,21}$

Many of the patients requiring ECMO tend to require prolonged mechanical ventilatory support, especially those on VV ECMO. These patients also have significantly compromised pulmonary compliance, and in some, very little or no tidal ventilation is feasible. However, with optimal control of carbon dioxide via ECMO, it is possible to minimize respiratory drive and awaken a patient. While some patients tolerate this well, others are intolerant of the endotracheal tube and find it difficult to spontaneously breathe, often resulting in being resedated. There may be a role for TT in these patients. However, PDT has not been commonly performed in ECMO patients due to a perceived lack of benefit or a perceived increase in the risk of bleeding, which may be caused by anticoagulation, underlying patient pathology, venous engorgement, and acquired von Willibrand deficiency in many of the patients on ECMO. ${ }^{22,23}$

There is minimal literature available regarding TT in ECMO and none assessing sedation requirements and associated benefits post-TT in patients on ECMO. Interestingly, pharmacokinetics of many drugs, including several sedative drugs, are markedly different with an ECMO circuit in situ. ${ }^{24,25}$ This is associated with conspicuously increased usage of sedative drugs during ECMO. ${ }^{26}$

Increased usage of fluids, sedation, vasopressors/inotropes, and blood have all been linked to worse outcomes in critically ill patients. These include an increase in mortality, risk of deep vein thrombosis, nursing workload, cost of care in ICU, and risk of arrhythmia, alongside worsening gas exchange and specific risks related to agents used such as propofol infusion syndrome. ${ }^{27-33} \mathrm{We}$ hypothesized that many of these adverse effects may be minimized by timely insertion of TT.

In this retrospective study we sought to assess whether (1) TT in adult patients on ECMO is associated with benefits of decreased use of analgesia, sedation, fluid usage including packed red cells (PRC), and vasopressor/inotrope requirements and (2) TT is associated with increased bleeding in adult patients on ECMO.

\section{Methods}

Setting

This retrospective observational study was conducted in a primarily cardiorespiratory ICU of a metropolitan teaching hospital in Australia. Ethics exemption was obtained from the hospital's Human Research and Ethics Committee (HREC/ 16/QPCH/303).

\section{Sample}

Medical records of all patients who received a TT while on ECMO from January 2011 to June 2016 were reviewed.

\section{Outcomes Measured}

Data included age, sex, primary diagnosis, type of ECMO support, number of days in ICU prior to ECMO, 5-day period pre-TT and 5 days post-TT, the number of days on ECMO altogether, amount of sedative drugs used, daily fluid balance, inotrope and vasopressor usage, any major complication of TT, and PRC transfusions received.

Based on the previously shown benefit of reduced sedation usage in intensive care patients post-TT, ${ }^{18}$ we chose sedation usage and other parameters on which sedation is likely to have an impact. All these parameters affect significant patient outcomes. Variables of interest included fluid intake, fluid output and balance, sedative drugs (propofol, midazolam, and dexmedetomidine), analgesic drugs (morphine and fentanyl), inotropes (dopamine, adrenaline, milrinone, and dobutamine), vasopressors (vasopressin, noradrenaline), and PRC usage. We analyzed PRC usage as a surrogate marker of significant bleeding requiring transfusion.

\section{Data Collection}

Data were collected for those patients who had a TT placed while on ECMO unless there was discontinuation of ECMO within 48 hours of placement of TT, in which case the patient was not included in the study. The decision for TT was made by the treating intensivist. The procedure was performed percutaneously in $100 \%$ of the cases in the ICU by 2 intensivists under direct bronchoscopic visualization. It was ensured that any coagulopathy present was corrected prior to starting procedure, and cross-matched blood and other blood products were readily available. The indication for TT varied among patients and most commonly included the need for prolonged ventilation and airway toileting with a view to also enable increased patient participation when able to tolerate more awake hours.

Anticoagulation was stopped early on the day of the TT, and coagulation profile and/or rotational thromboelastometry was 


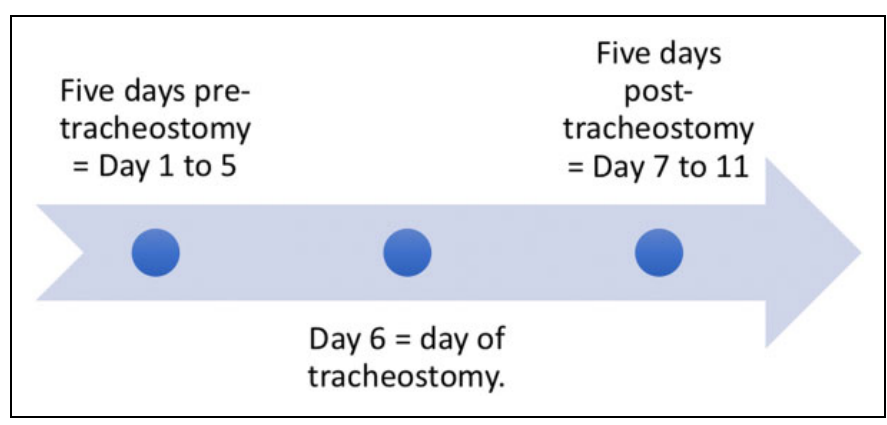

Figure I. Data were collected for 5 days pretracheostomy and 5 days posttracheostomy ignoring the values on the day of tracheostomy that was designated day 6 on the patient timeline. Data from day 6 (day of tracheostomy) were not included in the statistical analysis. Patients who were not supported with ECMO for the full I I days had missing values for the days they were off ECMO. ECMO indicates extracorporeal membrane oxygenation.

checked prior to TT. Coagulation abnormalities were corrected to goals set by treating intensivist.

Data were collected for 11 days in total, with 5 days pre-TT and 5 days post-TT. The date of TT placement was counted as "day 6." Data for "day 6" were not used (Figure 1). For patients who were provided both venoarterial (VA) ECMO and VV ECMO support, data were analyzed for the 5 days pre-TT and post-TT during their first TT period.

Patients who were not supported by ECMO for the full 11 days had missing values for the days for which ECMO had been withdrawn. Fluid balances were calculated for each 24-hour period starting from midnight. Sedation was calculated using 2 measures. One of the measures used midazolam and propofol (MP) converted to propofol equivalents in milligram. ${ }^{34}$ Since a large percentage of patients also received dexmedetomidine, a separate measure was used, which converted midazolam, propofol, and dexmedetomidine (MPD) to propofol equivalents Propofol in $\mathrm{mg}+\{(0.106 \times$ midazolam in $\mathrm{mg}) / 1.66\}+$ Dexmedetomidine/0.002\}. Due to total sedative dosages of MPD having a highly skewed distribution, this was log-transformed to enhance graphical presentation. Analgesic medications included morphine and fentanyl, for which doses were converted to morphine equivalents. ${ }^{35}$ Inotrope score (IS) was calculated using the formula: Dopamine dose $(\mu \mathrm{g} / \mathrm{kg} / \mathrm{min})$ + Dobutamine dose $(\mu \mathrm{g} / \mathrm{kg} / \mathrm{min})+100 \times$ Epinephrine dose $(\mu \mathrm{g} / \mathrm{kg} / \mathrm{min}) .{ }^{36}$ Vasopressor inotrope score (VIS) was calculated using the formula: IS $+10 \times$ Milrinone dose $(\mu \mathrm{g} / \mathrm{kg} / \mathrm{min})$ $+10000 \times$ Vasopressin dose $(\mathrm{U} / \mathrm{kg} / \mathrm{min})+100 \times$ Norepinephrine dose $(\mu \mathrm{g} / \mathrm{kg} / \mathrm{min}) .{ }^{36}$ The PRCs were counted as units, with each unit being 220 to $240 \mathrm{~mL}$. The volume of PRC transfused was measured as part of fluid balance (Figure 2).

Data were also collected for Sequential Organ Failure Assessment (SOFA) scores for all the patients for the first 4 days of their admission to the ICU and for the 24 hours prior to TT. This was used as a surrogate marker for severity of illness to check for a possible change in requirement of sedation, analgesia, vasopressor, and inotropes resulting from significant improvement or worsening in patient condition.

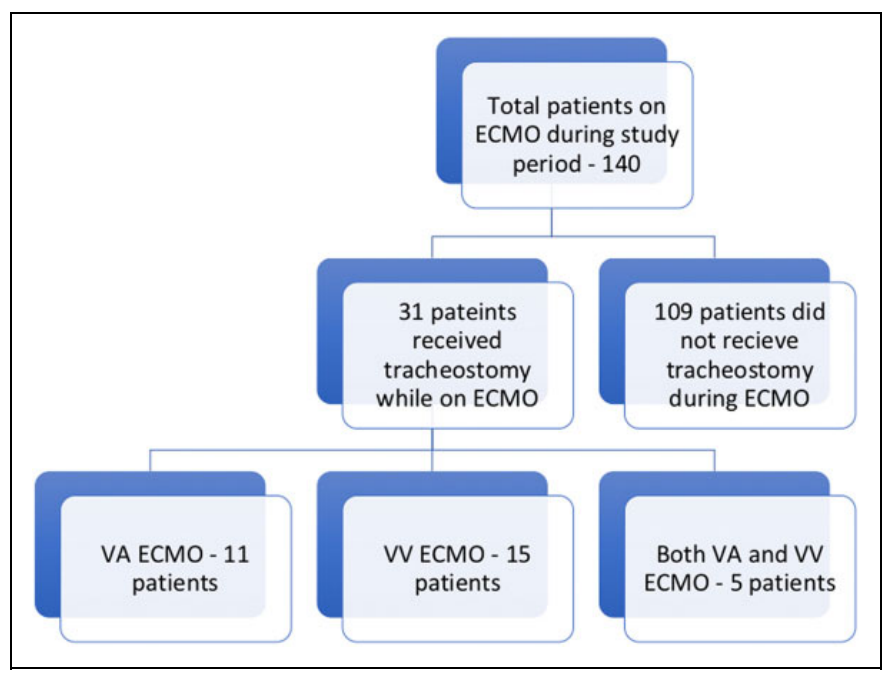

Figure 2. Patient selection during the study.

Table I. Demographics, ECMO, and Tracheostomy. ${ }^{a}$

\begin{tabular}{|c|c|}
\hline Male & 20 \\
\hline Female & II \\
\hline $\begin{array}{l}\text { Median time from admission to } \\
\text { tracheostomy, days }\end{array}$ & 7 (IQR 5-II) \\
\hline $\begin{array}{l}\text { Median time from ECMO initiation to } \\
\text { tracheostomy, days }\end{array}$ & 6 (IQR 4-9) \\
\hline $\begin{array}{l}\text { Total patients on VA ECMO with } \\
\text { tracheostomy }\end{array}$ & $16^{\mathrm{b}}$ \\
\hline Range, days & $1.9-85.9^{\mathrm{c}}$ \\
\hline $\begin{array}{l}\text { Total patients on VV ECMO with } \\
\text { tracheostomy }\end{array}$ & $20^{\mathrm{b}}$ \\
\hline Total duration in hours, days & 9205 (383.54) \\
\hline Mean duration in hours, days & $460.27(19.17)$ \\
\hline Range, days & $109-1990$ hours $(4.5-82.9)$ \\
\hline $\begin{array}{l}\text { Total ECMO days for patients with } \\
\text { tracheostomy }\end{array}$ & 561.8 \\
\hline Average days & $\begin{array}{c}\text { I8.I } \\
\text { Cardiogenic shock (5) } \\
\text { Cardiomyopathy (3) } \\
\text { Cardiac arrest (2) }\end{array}$ \\
\hline Underlying diagnosis for VA ECMO & $\begin{array}{c}\text { Other (I) } \\
\text { ARDS (7) } \\
\text { Pneumonia (4) } \\
\text { Pulmonary hemorrhage (I) }\end{array}$ \\
\hline Underlying diagnosis for VV ECMO & Other (3) \\
\hline
\end{tabular}

Abbreviations: ARDS, acute respiratory distress syndrome; ECMO, extracorporeal membrane oxygenation, IQR, interquartile range; $\mathrm{VA}$, venoarterial; $\mathrm{VV}$, venovenous.

${ }^{\mathrm{a}}$ For all patients, the first tracheostomy period while on VV or VA ECMO was analyzed.

${ }^{b}$ Five patients were on both VA ECMO and VV ECMO.

${ }^{\mathrm{C}}$ Four of the patients on VA ECMO were transferred to left ventricular assist device (LVAD) during their ICU stay.

\section{Statistical Analysis}

The distribution of variables was examined, and boxplots were obtained by day for each variable. Patient-level summary measures were derived for the pre-TT and post-TT periods. For 


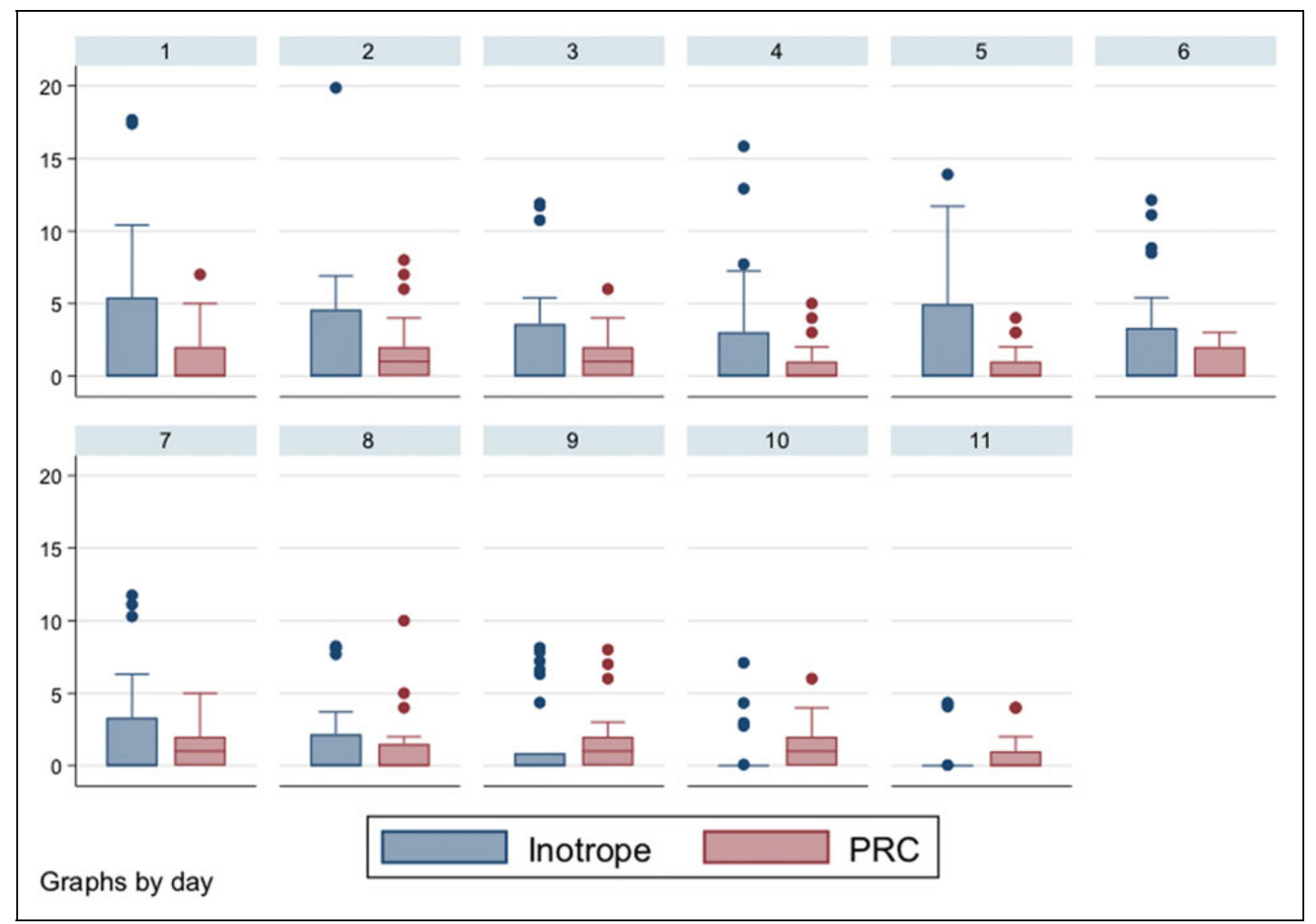

Figure 3. Boxplots of inotrope and PRC dose by day for patients with data. The center line in each box represents the median and the shaded area comprises the interquartile range (with the middle $50 \%$ of the observations). The whiskers (at the end of the middle line) denoted the range that contains $95 \%$ of the observations. Outliers are represented as dots. PRC indicates packed red cell.

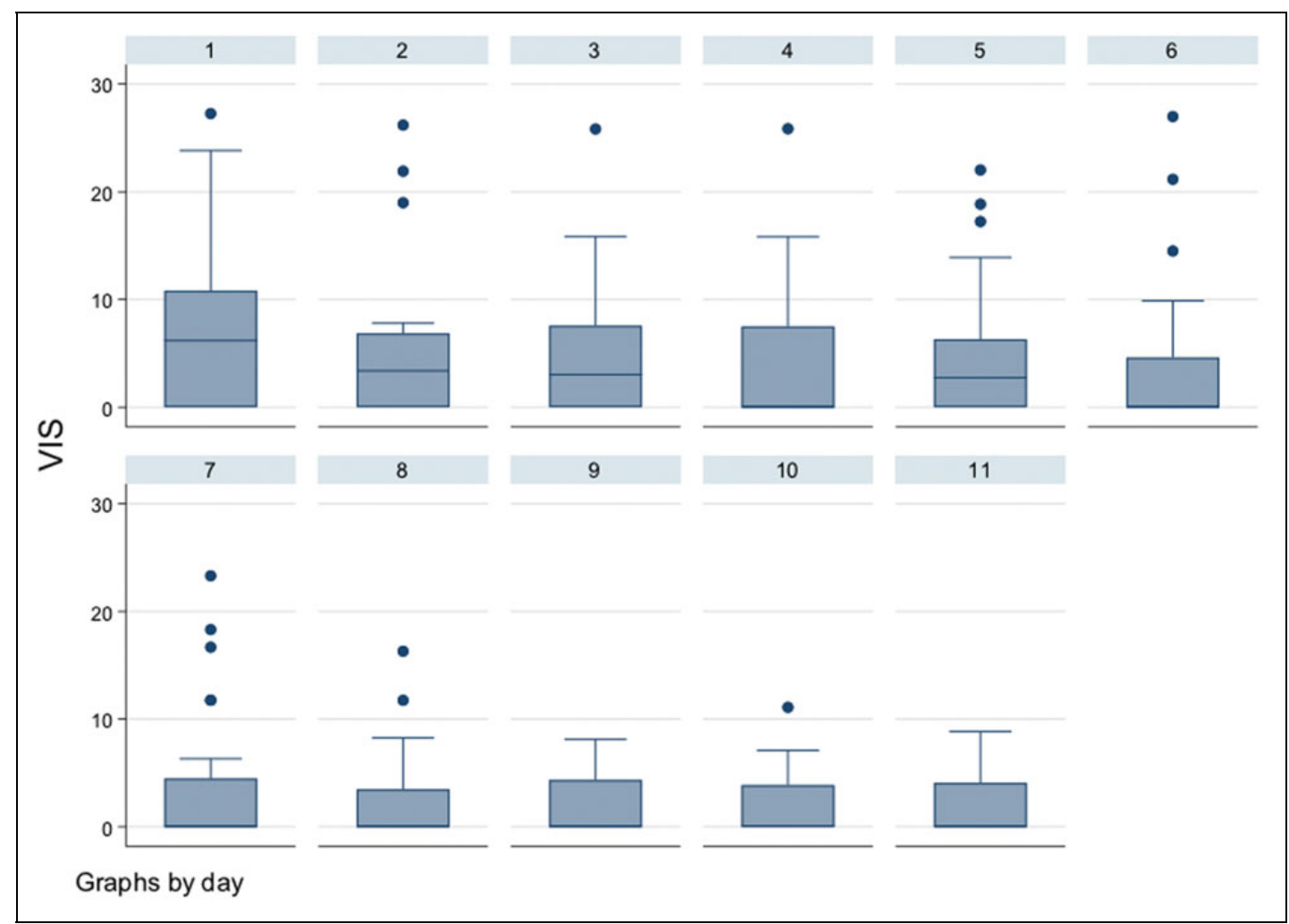

Figure 4. Boxplots of VIS by day for patients with data. The center line in each box represents the median and the shaded area comprises the interquartile range (with the middle $50 \%$ of the observations). The whiskers (at the end of the middle line) denoted the range that contains $95 \%$ of the observations. Outliers are represented as dots. VIS indicates vasopressor inotrope score. 


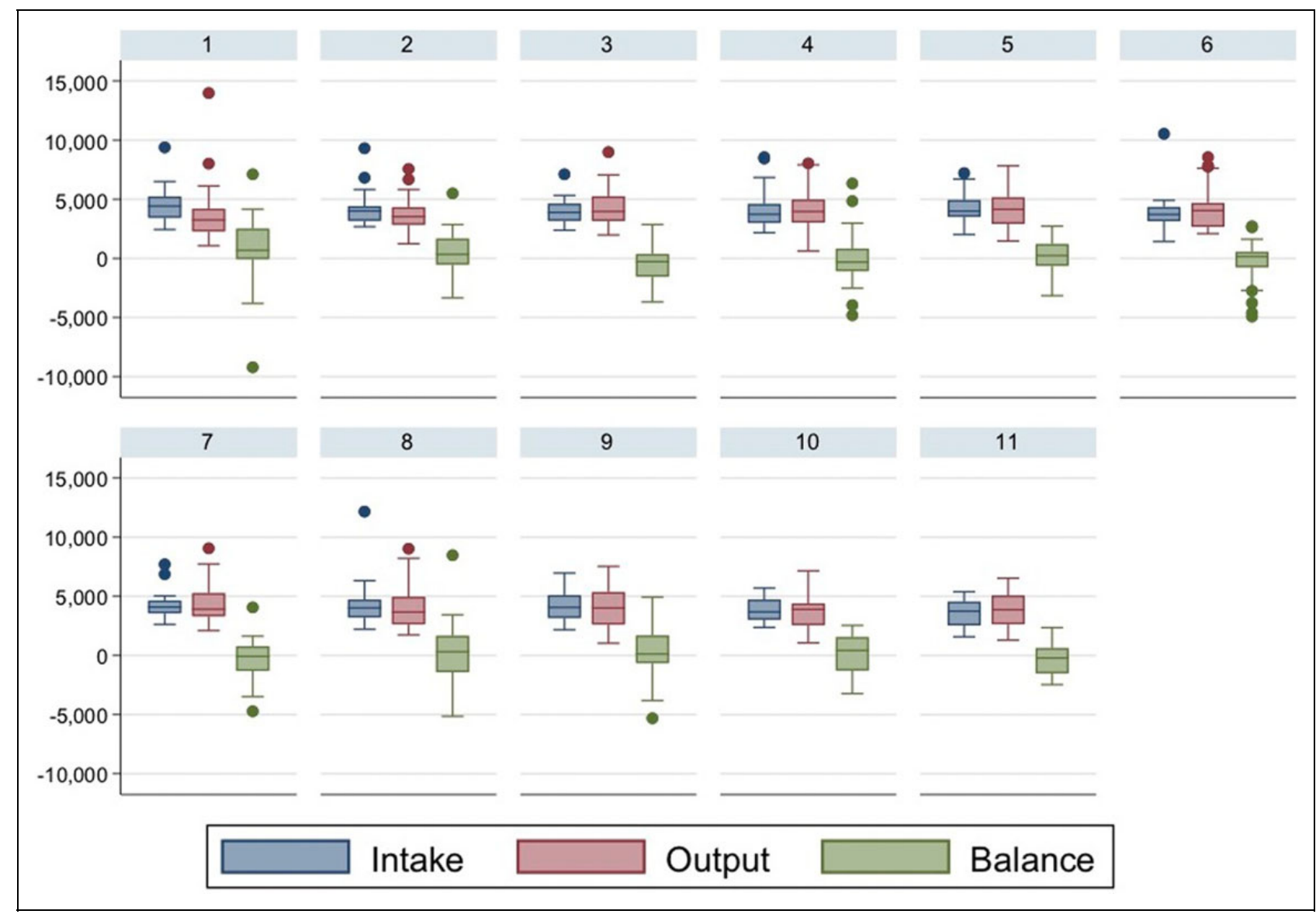

Figure 5. Boxplots of fluid intake, output, and balance by day for patients with data. Volume of packed red cells transfused was included in the fluid calculations. The center line in each box represents the median and the shaded area comprises the interquartile range (with the middle $50 \%$ of the observations). The whiskers (at the end of the middle line) denoted the range that contains $95 \%$ of the observations. Outliers are represented as dots.

variables that were normally distributed (ie, fluid intake, output, and balance) mean values were calculated for each patient for each period (pre-TT and post-TT). For variables with a skewed distribution median values (pre-TT and post-TT) were used as the measure of interest. The mean values of the pre-TT and post-TT fluid variables (ie, patient-level means) were compared using paired $t$ tests. The distribution of values for pre-TT and post-TT variables with skewed distributions was compared using Wilcoxon matched-pairs signed-rank test. Analyses were performed using the Stat Statistical Software Package (version 15).

\section{Results}

A total of 140 adult patients were supported by ECMO from January 1, 2011, to June 30, 2016. Of these patients, 31 (22\%) received a TT while on ECMO. Of these, 15 received VV ECMO, 11 were placed on VA ECMO, and 5 patients were on both VA and VV ECMO during their ICU stay. Total number of days on ECMO for these 31 patients was 561.8 with a mean of 18.1 days. Total VA ECMO days were 151 with a mean duration of 13.1 days. For VV ECMO, 340 days were spent on ECMO with average of 22.7 days per patient. Median duration of time from admission to TT placement was 7 days, and median duration from initiation of ECMO and TT placement was 6 days.

Seven patients had data missing for 1 or more of the 5 days prior to placement of TT and 8 patients had data missing in 1 or more of the 5 days after placement of TT. Missing data occurred due to either patients having less than 5 days between initiation of ECMO and placement of TT (pre-TT period) or being weaned off ECMO sooner than 5 days post-TT insertion (post-TT period). The underlying causes for placement of both VA ECMO and VA ECMO are included in the attached Table 1.

The distribution of IS ( $P$ value .01$)$ and VIS ( $P$ value .01$)$ differed between pre-TT and post-TT periods and showed a substantial reduction in post-TT period. Although 13 of the 31 patients did not receive inotropes, and 6 patients had no inotropic or vasopressor usage during the period assessed (ie, value $=0$ ), the interquartile range is larger in pre-TT compared to post-TT, as shown in Figures 3 and 4.

The overall mean daily fluid intake was $4157 \mathrm{~mL}$, output was $4003 \mathrm{~mL}$, and fluid balance had a mean of $154 \mathrm{~mL}$ 


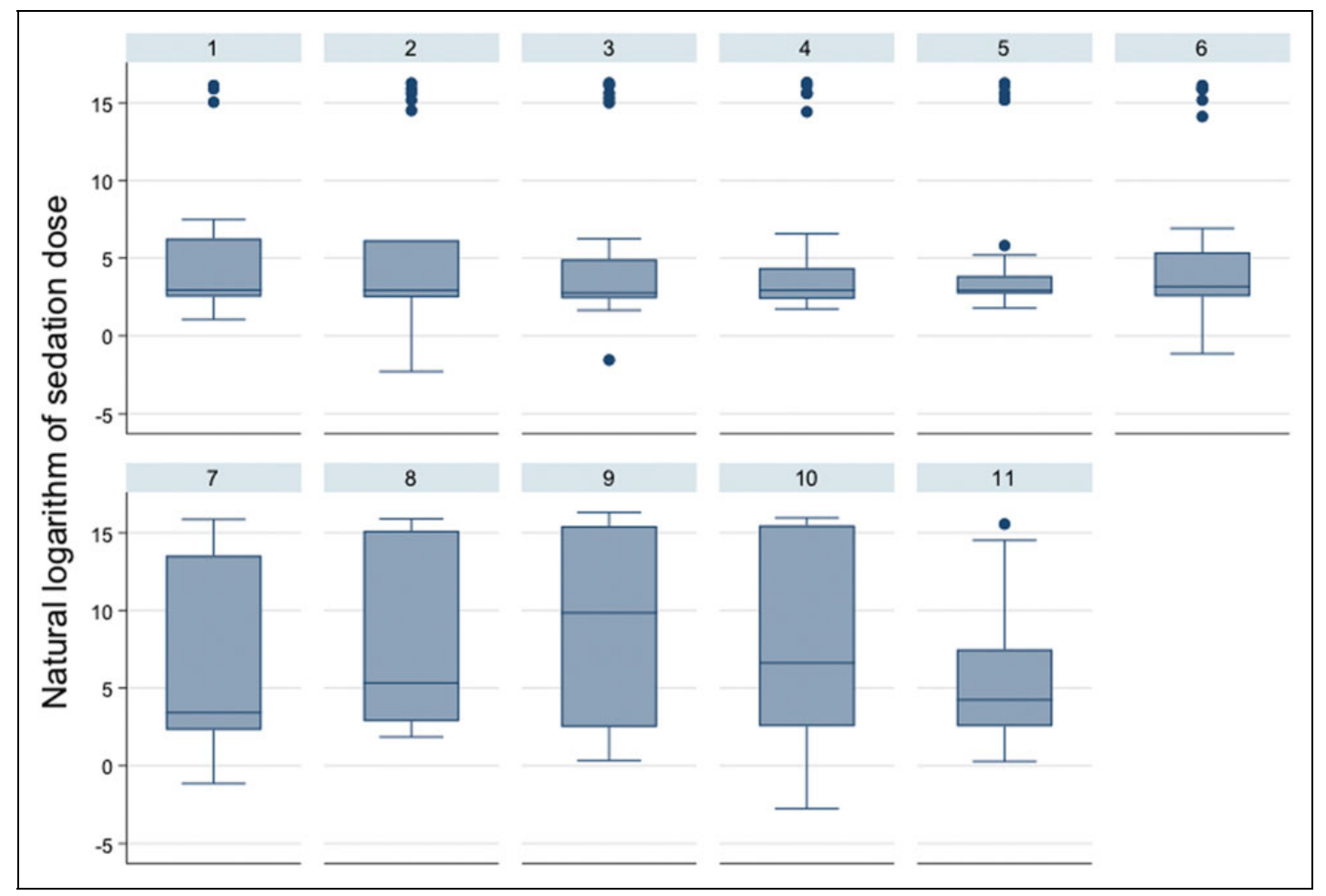

Figure 6. Boxplots of total sedative (MPD) dose on the logarithmic scale by day for patients with data. The center line in each box represents the median and the shaded area comprises the interquartile range (with the middle $50 \%$ of the observations). The whiskers (at the end of the middle line) denoted the range that contains $95 \%$ of the observations. Outliers are represented as dots. MPD indicates midazolam, propofol, and dexmedetomidine.

positive. These did not differ significantly between periods defining pre-TT and post-TT ( $P$ value $.69, .65$, and .55 for input, output, and fluid balance, respectively; Figure 5).

The overall median sedative dose was 18 units, while the interquartile range (10-783) reflects the large amount of variability and the highly skewed distribution for this variable. This is particularly evident for the post-TT interquartile range (6-2 000000 ). Sedative dose ( $P$ value .54 and .31 for the 2 measures of sedation, MP and MPD) and PRC ( $P$ value .46$)$ did not differ significantly between periods defining pre-TT and post-TT. There was a trend toward reduction in analgesia usage, which did not reach statistical significance $(P$ value .1$)$ as shown in Figures 6 and 7 and Table 2.

Differences between mean SOFA scores on days 1 to 4 from day of admission were compared to values during the 24-hour period prior to placement of TT ( 1 day pre-TT) using a paired $t$ test. Mean values on each day and averaged over 4 days (days 1-4) are shown in Table 3 along with the results of significance tests. Overall SOFA scores ranged from 3 to 20. Mean scores were significantly lower on days 2,3 , and 4 compared to scores during the day pre-TT. The difference between mean values on day 1 (mean: 9.4; standard deviation [SD] 3.5) and day pre-TT (mean: 10.6; SD 3.1) did not reach statistical significance.

A3 (APACHE III) scores ranged from 46 to 156 . The median $\mathrm{A} 3$ score was 71 (interquartile range: 57-97).

\section{Discussion}

This is the first study reporting the safety of TT placement and its putative benefits in patients on ECMO. There is increasing awareness of the possibility and potential benefits of early mobilization and rehabilitation of ECMO patients where possible. ${ }^{37,38}$ Therefore, the risk and benefits of strategies such as early extubation versus TT placement to enable the same need scrutiny.

This study confirmed that TT can be safely performed in ECMO patients. There was no increase in PRC use in tracheostomized patients. Use of PRC in our patients undergoing TT did not differ significantly in the pre-TT and post-TT period, suggesting there were no major bleeding events. We used this as a surrogate for safety of TT. On review of all charts, no other major adverse events such as loss of airway, major airway bleeding, arrhythmias, worsening of ventilation, and so on were noted. A previous study examining complications of TT in patients on ECMO also reached a similar conclusion. ${ }^{39}$ However, hemostasis can become a challenge if a major complication is encountered and the risk and benefits of TT need to be carefully examined on a case-by-case basis. Especially, patients on VV ECMO who are likely to have a prolonged ECMO run and cannot be extubated may be considered for TT once they are stabilized on ECMO. Considering the risks, it is important that attempts be made to wean sedation and to assess suitability for extubation prior to performing a TT, as in general critically ill patients. 


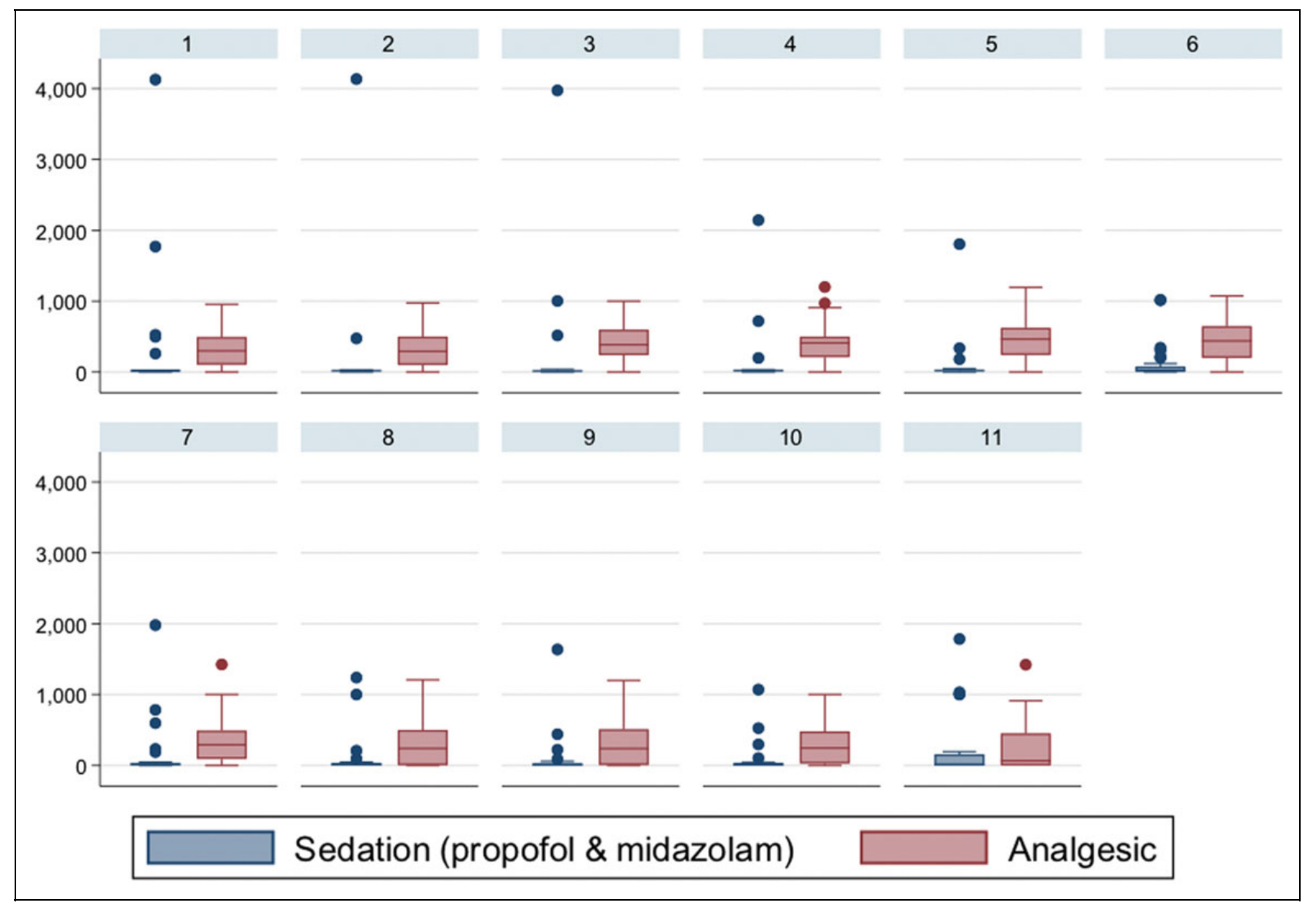

Figure 7. Boxplots of sedation dose (MP) and analgesic dose by day for patients with data. The center line in each box represents the median and the shaded area comprises the interquartile range (with the middle $50 \%$ of the observations). The whiskers (at the end of the middle line) denoted the range that contains $95 \%$ of the observations. Outliers are represented as dots. MP indicates midazolam and propofol.

Table 2. Summary Statistics for Patient-Level Summary Measures Pre- and Posttracheostomy Placement. ${ }^{\text {a }}$

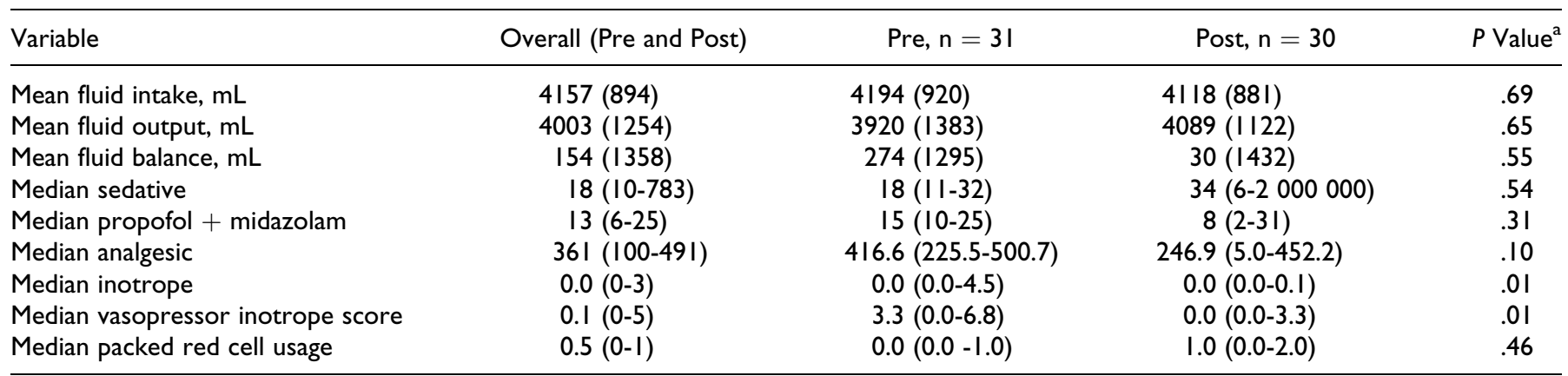

${ }^{\text {a }}$ Statistics are mean (standard deviation) with $P$ values from paired $t$ test or median (interquartile range) with $P$ values from Wilcoxon signed-rank test.

Interestingly, although the use of vasopressors and inotropes showed a significant reduction in post-TT period, this was not associated with a difference in use of sedation. Clearly, a decrement in vasoactive drug use is desirable as an increased requirement is usually associated with increased fluid administration and inability to minimize extravascular lung water whether by use of diuretics or ultrafiltration. Fluid balance, along with usage and output, was not found to be different before and after TT insertion.
Though not clearly explained without an associated reduction in sedation, this is an important outcome and needs to be explored further with a larger sample size. Possible reasons for this may be related to a reduction in sedation postTT, which failed to reach the prespecified significance level, especially with wide variation in sedation usage. A larger study to assess the differences specifically for a longer period of pre-TT and post-TT follow-up may clarify this hypothesis generating finding. 
Table 3. Comparison of SOFA Scores from Days I to 4 of Admission Compared to Day Prior to Tracheostomy. ${ }^{\text {a }}$

\begin{tabular}{|c|c|c|c|c|c|}
\hline \multirow[b]{2}{*}{ SOFA Scores } & \multicolumn{2}{|c|}{ Comparison Day } & \multicolumn{2}{|c|}{ Day Pre-TT } & \multirow[b]{2}{*}{$P$ Value } \\
\hline & Mean & SD & Mean & SD & \\
\hline Day I & 9.4 & 3.5 & 10.6 & 3.1 & .0612 \\
\hline Day 2 & 9.1 & 3.6 & 10.6 & 3.1 & .0152 \\
\hline Day 3 & 8.6 & 3.9 & 10.6 & 3.1 & .0065 \\
\hline Day 4 & 8.2 & 3.6 & 10.6 & 3.1 & .0003 \\
\hline Mean (days I-4) & 8.8 & 3.4 & 10.6 & 3.1 & .004 \\
\hline
\end{tabular}

Abbreviations: SD, standard deviation; SOFA, Sequential Organ Failure Assessment; TT, tracheostomy.

${ }^{a}$ Days I to 4 on this table refer to days on admission to intensive care. This is compared to the SOFA scores calculated for the day prior to tracheostomy.

There was no significant difference $(P$ value .06$)$ in the severity of illness of patients between the day of admission and 24-hour period prior to TT placement as assessed by SOFA scores. However, the patients had significantly greater SOFA scores on day before TT when compared to days 2,3 , and 4 , indicating that the reduction in vasopressor and inotrope doses was not related to resolution of illness itself and may at least in part be attributed to placement of a TT.

Although there was no statistically significant difference in the use of sedation or analgesia, there was a trend toward reduction in analgesia usage, and it would be interesting to see if the trend became stronger with a larger sample size. Lack of a significant sedation difference could be because of inability to reduce sedation due to various ongoing clinical indications unrelated to TT. Fear of ECMO cannula movement is one such common reason, as dislodgement could be catastrophic.

Placement of a TT (instead of an endotracheal tube) may have other additional benefits for the patient and clinician who were not investigated as part of this study, including improved patient communication, rehabilitation participation, mobilization, easier access to tracheobronchial tree for toileting and suctioning, and reduced sedation. Larger studies with longer follow-up may help in identifying a difference in these parameters for patients on ECMO.

This single-center study does suffer from its size, but it highlights some important findings that are of relevance to the evolving practice of ECMO. Blinding of clinicians to sedative and analgesic usage was not possible in this retrospective study. It is reassuring that complications of TT can be minimized when risk and benefits are acceptable. In addition, the potential for reduction in requirements of vasopressors and inotropes is physiologically significant. A larger sample size may clarify this further along with identifying many other patient-centered benefits of performing a TT in ECMO patients.

\section{Conclusions}

Tracheostomy can be safely performed in selected ECMO patients in who benefits outweigh the risks without an associated increase in use of PRC. Performing a TT for patients while they are still requiring ECMO support may result in reduction in inotropic or vasopressor usage although this needs to be further assessed in a larger study. Similarly, there may be a benefit in terms of reduction in analgesic usage, which may be seen in a study with larger sample size.

\section{Acknowledgments}

We acknowledge Dr Toni Kinneally (MBBS student at the time of study) and Ms Stephanie Fisquet (pharmacist, The Prince Charles Hospital) for their assistance in data collection and conversion factors for sedation and analgesia usage. We recognize Dr Karen Hay for running the statistical analysis for this study. We acknowledge the great care provided to these ECMO patients by the entire multidisciplinary team at The Prince Charles hospital Intensive Care Service.

\section{Authors' Note}

Jatinder Grewal, Anna-Liisa Sutt, Kiran Shekar, and John Fraser contributed to conception and design, data analysis, and interpretation. George Cornmell gave administrative support. All authors provided study materials or patients, collected and assemble data, wrote manuscript, and gave final approval of manuscript.

\section{Declaration of Conflicting Interests}

The author(s) declared no potential conflicts of interest with respect to the research, authorship, and/or publication of this article.

\section{Funding}

The author(s) disclosed receipt of the following financial support for the research, authorship, and/or publication of this article: This study was supported by NHMRC CRE ACTIONS.

\section{References}

1. Shekar K, Mullany DV, Thomson B, Ziegenfuss M, Platts DG, Fraser JF. Extracorporeal life support devices and strategies for management of acute cardiorespiratory failure in adult patients: a comprehensive review. Crit Care. 2014;18(3):219.

2. Butt W, MacLaren G. Extracorporeal membrane oxygenation 2016: an update. [version 1; referees: 3 approved]. F1000Research 2016;5(F1000 Faculty Rev):750.

3. Shekar K, Brodie D, Fraser J, MacLaren G. Appraising extracorporeal life support-current and future roles in adult intensive care. Crit Care Resusc. 2017;19(suppl 1):5-7.

4. Australia and New Zealand Extracorporeal Membrane Oxygenation (ANZ ECMO) Influenza Investigators, Davies A, Jones D, Bailey M, et al. Extracorporeal membrane oxygenation for 2009 influenza A (H1N1) acute respiratory distress syndrome. JAMA. 2009;302(17):1888-1895.

5. Peek GJ, Mugford M, Tiruvoipati R, et al. Efficacy and economic assessment of conventional ventilatory support versus extracorporeal membrane oxygenation for severe adult respiratory failure (CESAR): a multicentre randomised controlled trial. Lancet. 2009;374(9698):1351-1363.

6. Smith M, Vukomanovic A, Brodie D, Thiagarajan R, Rycus P, Buscher H. Duration of veno-arterial extracorporeal life support (VA ECMO) and outcome: an analysis of the Extracorporeal Life Support Organization (ELSO) registry. Crit Care. 2017;21(1):45. 
7. Combes A, Hajage D, Capellier G, et al. Extracorporeal membrane oxygenation for severe acute respiratory distress syndrome. N Engl J Med. 2018;378(21):1965-1975.

8. Goligher EC, Tomlinson G, Hajage D, et al. Extracorporeal membrane oxygenation for severe acute respiratory distress syndrome and posterior probability of mortality benefit in a post hoc Bayesian analysis of a randomized clinical trial. JAMA. 2018;320(21): 2251-2259.

9. Camporota L, Nicoletti E, Malafronte M, et al. International survey on the management of mechanical ventilation during ECMO in adults with severe respiratory failure. Minerva Anestesiol. 2015;81(11):1170-1183.

10. Mohite PN, Sabashnikov A, Reed A, et al. Extracorporeal life support in "awake" patients as a bridge to lung transplant. Thorac Cardiovasc Surg. 2015;63(8):699-705.

11. Shekar K, Roberts JA, Ghassabian S, et al. Sedation during extracorporeal membrane oxygenation-why more is less. Anaesth Intensive Care. 2012;40(6):1067-1069.

12. Andriolo BN, Andriolo RB, Saconato H, Atallah ÁN, Valente O. Early versus late tracheostomy for critically ill patients. The Cochrane Database of Systematic Reviews. 2015;1:Cd007271.

13. Young D, Harrison DA, Cuthbertson BH, Rowan K, TracMan C. Effect of early vs late tracheostomy placement on survival in patients receiving mechanical ventilation: the TracMan randomized trial. JAMA. 2013;309(20):2121-2129.

14. Groves DS, Durbin CG Jr. Tracheostomy in the critically ill: indications, timing and techniques. Curr Opin Crit Care. 2007; 13(1):90-97.

15. Durbin CG Jr. Tracheostomy: why, when, and how? Respir Care. 2010;55(8):1056-1068.

16. Kluge S, Baumann HJ, Maier C, et al. Tracheostomy in the intensive care unit: a nationwide survey. Anesth Analg. 2008;107(5): 1639-1643.

17. Vargas M, Sutherasan Y, Antonelli M, et al. Tracheostomy procedures in the intensive care unit: an international survey. Crit Care. 2015;19:291.

18. Nieszkowska A, Combes A, Luyt CE, et al. Impact of tracheotomy on sedative administration, sedation level, and comfort of mechanically ventilated intensive care unit patients. Crit Care Med. 2005;33(11):2527-2533.

19. Arabi Y, Haddad S, Shirawi N, Al Shimemeri A. Early tracheostomy in intensive care trauma patients improves resource utilization: a cohort study and literature review. Crit Care. 2004;8(5): R347-R352.

20. Sutt AL, Cornwell P, Mullany D, Kinneally T, Fraser JF. The use of tracheostomy speaking valves in mechanically ventilated patients results in improved communication and does not prolong ventilation time in cardiothoracic intensive care unit patients. $J$ Crit Care. 2015;30(3):491-494.

21. Sutt AL, Fraser JF. Patients want to be heard-loud and clear! Crit Care. 2017;21(1):6.

22. Heilmann C, Geisen U, Beyersdorf F, et al. Acquired von Willebrand syndrome in patients with extracorporeal life support (ECLS). Intensive Care Med. 2012;38(1):62-68.

23. Jones MB, Ramakrishnan K, Alfares FA, et al. Acquired von Willebrand syndrome: an under-recognized cause of major bleeding in the cardiac intensive care unit. World J Pediatr Congenit Heart Surg. 2016;7(6):711-716.

24. Shekar K, Roberts JA, Mullany DV, et al. Increased sedation requirements in patients receiving extracorporeal membrane oxygenation for respiratory and cardiorespiratory failure. Anaesth Intensive Care. 2012;40(4):648-655.

25. Shekar K, Fraser JF, Smith MT, Roberts JA. Pharmacokinetic changes in patients receiving extracorporeal membrane oxygenation. J Crit Care. 2012;27(6):741.e9-741.e18.

26. Nigoghossian CD, Dzierba AL, Etheridge J, et al. Effect of extracorporeal membrane oxygenation use on sedative requirements in patients with severe acute respiratory distress syndrome. Pharmacotherapy. 2016;36(6):607-616.

27. Barrientos-Vega R, Sanchez-Soria MM, Morales-Garcia C, Robas-Gomez A, Cuena-Boy R, Ayensa-Rincon A. Prolonged sedation of critically ill patients with midazolam or propofol: impact on weaning and costs. Crit Care Med. 1997;25(1):33-40.

28. Boyd JH, Forbes J, Nakada TA, Walley KR, Russell JA. Fluid resuscitation in septic shock: a positive fluid balance and elevated central venous pressure are associated with increased mortality. Crit Care Med. 2011;39(2):259-265.

29. Girard TD, Kress JP, Fuchs BD, et al. Efficacy and safety of a paired sedation and ventilator weaning protocol for mechanically ventilated patients in intensive care (Awakening and Breathing Controlled trial): a randomised controlled trial. Lancet. 2008; 371(9607):126-134.

30. Jackson DL, Proudfoot CW, Cann KF, Walsh T. A systematic review of the impact of sedation practice in the ICU on resource use, costs and patient safety. Crit Care. 2010;14(2):R59.

31. Krajcova A, Waldauf P, Andel M, Duska F. Propofol infusion syndrome: a structured review of experimental studies and 153 published case reports. Crit Care. 2015;19:398.

32. Schmidt M, Bailey M, Kelly J, et al. Impact of fluid balance on outcome of adult patients treated with extracorporeal membrane oxygenation. Intensive Care Med. 2014;40(9):1256-1266.

33. Shehabi Y, Bellomo R, Reade MC, et al. Early intensive care sedation predicts long-term mortality in ventilated critically ill patients. Am J Respir Crit Care Med. 2012;186(8):724-731.

34. Devlin JW, Roberts RJ. Pharmacology of commonly used analgesics and sedatives in the ICU: benzodiazepines, propofol, and opioids. Anesthesiol Clin. 2011;29(4):567-585.

35. Woodhouse A, Hobbes AF, Mather LE, Gibson M. A comparison of morphine, pethidine and fentanyl in the postsurgical patientcontrolled analgesia environment. Pain. 1996;64(1):115-121.

36. Gaies MG, Gurney JG, Yen AH, et al. Vasoactive-inotropic score as a predictor of morbidity and mortality in infants after cardiopulmonary bypass. Pediatr Crit Care Med. 2010;11(2):234-238.

37. Abrams D, Javidfar J, Farrand E, et al. Early mobilization of patients receiving extracorporeal membrane oxygenation: a retrospective cohort study. Crit Care. 2014;18(1):R38.

38. Ko Y, Cho YH, Park YH, et al. Feasibility and safety of early physical therapy and active mobilization for patients on extracorporeal membrane oxygenation. ASAIO J. 2015;61(5):564-568.

39. Braune S, Kienast S, Hadem J, et al. Safety of percutaneous dilatational tracheostomy in patients on extracorporeal lung support. Intensive Care Med. 2013;39(10):1792-1799. 